\title{
Effects of wearing a pressure redistribution belt while lying in a hospital bed
}

\author{
François Fraysse ${ }^{\mathrm{a},{ }^{*}}$, Nathan Daniell ${ }^{\mathrm{a}}$ and Gunther Paul ${ }^{\mathrm{a}}$ \\ ${ }^{a}$ Ergolab, Mawson Institute, University of South Australia, Mawson Lakes SA 5095
}

\begin{abstract}
Bedsores (ulcers) are caused by multiple factors which include, but are not limited to; pressure, shear force, friction, temperature, age and medication. Specialised support services, such as specialised mattresses, sheepskin coverings etc., are thought to decrease or relieve pressure, resulting in a lowering of pressure ulcer incidence [3]. The primary aim of this study was to compare the upper/central body pressure distribution between normal lying in a hospital bed versus the use of a pressure redistribution belt. The study involved 16 healthy voluntary subjects lying on a hospital bed with and without wearing the belt. Results showed that the use of a pressure redistribution belt results in reduced pressure peaks and prevents the pressure from increasing over time.
\end{abstract}

Keywords: pressure ulcers, bedsores, pressure mapping, pressure redistribution

\section{Introduction}

Bedsores (ulcers) are caused by multiple factors which include, but are not limited to; pressure, shear force, friction, temperature, age and medication. Bedsores are a significant medical issue and can be fatal. One of the primary causes of bedsores is extended periods of pressure. This risk often occurs in a hospital or nursing home setting where patients are required to lie in a bed for an extended period of time. The compression caused by the force of the bones against the supporting surface for this extended period of time, with reduced tissue perfusion, will restrict blood supply. The restriction of blood supply may result in necrosis which causes a bedsore [2].

Previous studies have shown that pressure ulcers are associated with peak pressures [1]. Peak pressures are typically manifested in regions of bone protuberance (e.g. ischial tuberosities).

One of the primary methods used in the prevention or treatment of a bedsore is to relieve pressure [2]. Specialised support services, such as specialised mattresses, sheepskin coverings etc., are thought to decrease or relieve pressure resulting in a lowering of pressure ulcer incidence when compared with standard hospital mattresses [3].
Remo Di Sotto has developed a portable belt ('RemoBelt') that provides protection to areas of the hips and coccyx that are susceptible to serious pressure sores and ulceration. The primary aim of this study was to compare the upper/central body pressure distribution between normal lying in a hospital bed versus the use of a pressure redistribution belt. This study measured the average pressure, contact area and pressure distribution to quantify any differences that may have occurred.

\section{Material and methods}

\subsection{Subjects}

Sixteen healthy subjects ( 8 male, 8 female; age $28 \pm 9$ yrs., stature $171 \pm 7 \mathrm{~cm}$, body mass $69 \pm 11 \mathrm{~kg}$ ) were recruited from the Mawson Institute at UniSA. Prior to each session the subjects were provided with an information sheet and signed a consent form. All subjects wore light clothing with empty pockets (no wallet, coins, etc) during data collection. This minimised any impact on the pressure measurements by clothes.

\footnotetext{
*Corresponding author. E-mail: francois.fraysse@unisa.edu.au. Phone: (+61) 883023628
} 


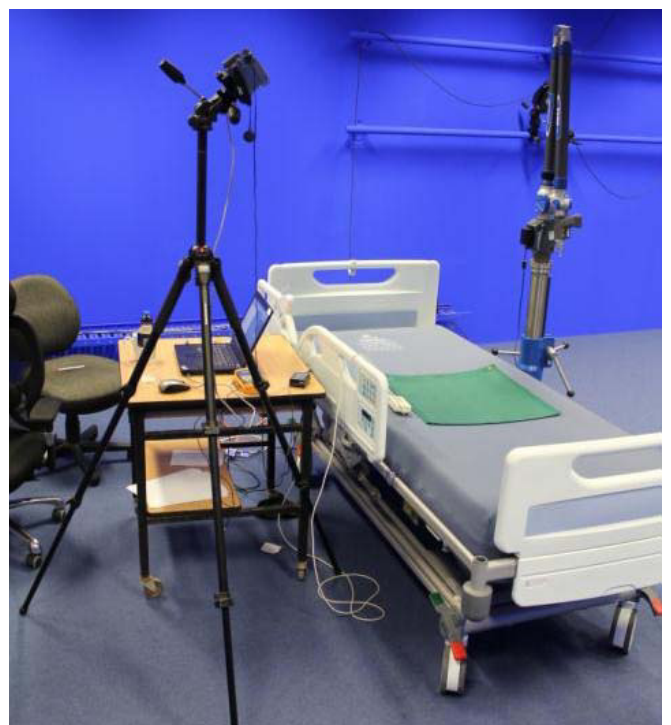

Fig. 1. Experimental set-up.

\subsection{Test sequence}

The data collection was completed on 8 different days over a period of two weeks. In total, 32 sessions were conducted (16x test afternoon and 16x re-test morning). All trials in the afternoon session were conducted between 12 p.m. and 4 p.m. with 8 trials per session, per subject. This was repeated for the morning session where all trials were completed between 8 a.m. and 12 p.m. This resulted in a total of 256 trials being completed during the data collection phase of this study (16 subjects $\times 8$ tests $\times 2$ repetitions).

The eight trials completed with each subject for each session were:

- Baseline: Unsupported lying on side and back

- Case 1\&2: belt supported lying using a size 1 belt, on side \& back

- $\quad$ Case 3\&4: belt supported lying using a size 2 belt, on side \& back

- Case 5\&6: belt supported lying using a size 3 belt, on side \& back

'Size 1' corresponds to the smallest belt and 'Size 3 ' to the largest. For each trial the subject was asked to lie on the bed in the desired position (side or back) without moving for 5 minutes. Pressure was recorded for 4 minutes for each trial using the Tekscan CONFORMat ${ }^{\circledR}$ system. The pressure mat recorded pressure with 1024 (32x32) individual sensors (Fig. 1). The sampling frequency was set at $2.5 \mathrm{~Hz}$ so 600 frames recorded for each trial. The experimental setup is shown on Fig. 1.

The subjects were required to get out of the hospital bed when changing the size of the belt. This ensured the belt was fitted correctly for each of the trials.

\subsection{Data processing}

\subsubsection{Average pressure}

For each trial, an average pressure $\mathrm{P}_{\mathrm{AVG}}(\mathrm{t})$ was calculated at each frame $t$, as the average pressure over the contact area (sensors showing a pressure of 0 were ignored). Then the average pressure $\mathrm{P}_{\mathrm{AVG}}$ for the whole trial was determined as the average of $\mathrm{P}_{\mathrm{AVG}}(\mathrm{t})$ over time, where $\mathrm{N}$ is the number of measured frames $(600)$ :

$$
\mathrm{P}_{\mathrm{AVG}}=\frac{1}{N} \sum_{t=1}^{N} P_{A V G}(t)
$$

\subsubsection{Normalisation}

To allow comparison between subjects, the pressure values were normalised. Normalisation was defined so that the average pressure $\mathrm{P}_{\mathrm{AVG}}$ was 1 for the trial where no belt was used, and the subject was lying on his/her back:

$$
\begin{aligned}
& \mathrm{P}_{\mathrm{AVGN}}=\frac{P_{A V G}}{P_{A V G}^{0}} \\
& \mathrm{P}_{\mathrm{AVGN}}(t)=\frac{P_{A V G}(t)}{P_{A V G}^{0}}
\end{aligned}
$$

$\mathrm{P}_{\mathrm{Avg}}{ }^{0}$ is the average pressure from the trial where no belt was used, and subjects were lying on their back. The average and standard deviations (SD) were then calculated across the 16 subjects.

\subsubsection{Contact area}

For the contact area, a similar methodology was employed. First, the contact area for each frame was determined by selecting the sensors showing a nonzero pressure. The area of a sensor is $2.17 \mathrm{~cm}^{2}$. The average contact area over time was then calculated for each trial, and the average and standard deviation over the 16 subjects were computed. No normalisation was used for the contact area values. 


\subsubsection{Pressure distribution}

In order to identify pressure peaks and have a general idea of the pressure distribution, the following method was employed.

First the pressure for each sensor at each frame was normalised by the average pressure $\mathrm{P}_{\mathrm{Avg}}$ for the corresponding trial (Equation 3). (Note that in this case the pressure is normalised independently for each trial).

Twenty-five pressure groups were defined, corresponding to a range of 0 to 5 times the average pressure $\mathrm{P}_{\mathrm{Avg}}$; the first group therefore corresponds to pressures between 0 and 0.2 times the average, the second category from 0.2 to 0.4 the average pressure, etc. For each group, the percentage of the contact area where the pressure is comprised in this group was then calculated. Finally, the histogram graph showing the percentage of contact area versus the normalised pressure groups was plotted.

This histogram plot shows the distribution of pressure: if for instance the pressure is perfectly even, then the histogram will show only one peak for the $0.8-1$ category, corresponding to $100 \%$ of the contact area. With increasingly distributed pressure, the histogram will be more stretched and spread. A histogram stretched towards the right indicates the existence of pressure peaks (small areas of high pressure). ferences between the morning and afternoon sessions ( $p<0.05$ for all cases). Therefore, results from the afternoon sessions have been presented in this paper.

\subsection{Effect of position}

Fig. $2 b$ shows the comparison between averaged normalised pressures on the contact area, when no belt was used for subjects lying on their back and side. Note that the normalised average value is 1 for the former case because of the definition of the normalised pressure values. The position has a significant effect on the average pressure $(\mathrm{p}<0.001)$. Fig. $2 \mathrm{a}$ provides an example of the differences in pressure distribution between the subject lying on their back or side.

\subsection{Effect of belt on average pressure}

Fig. 3 and Table 1 show the average normalised pressures $\mathrm{P}_{\mathrm{AVGN}}$ without using the belt, and when using a belt. The value presented for the latter case is the average for the 3 belts used. Results show that the use of a belt significantly increases the average pressure, both when lying on the back $(\mathrm{p}<0.01)$ and on the side $(p<0.01)$. When using a belt, the position has a significant effect $(p<0.01)$ on the average pressure.

\section{Results}

\subsection{Effect of time of the day}

To evaluate the influence of the time of the day, the average pressures for each trial were compared for each subject. Results showed no significant dif-

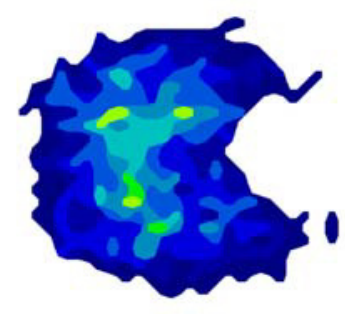

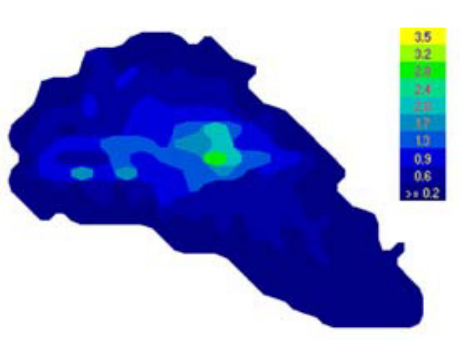

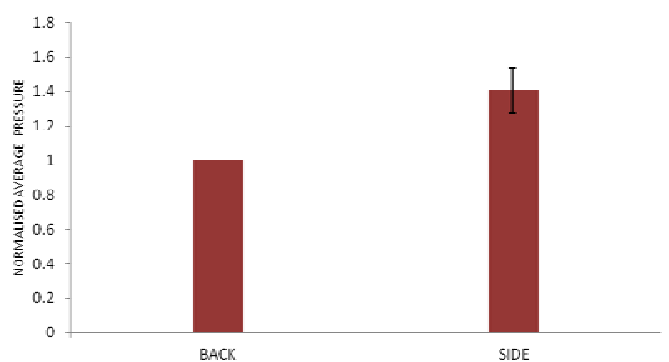

Fig. 2. a. Typical normalized pressure distribution pattern without belt, with subject lying on the back (left) and on the side (right). b. Comparison of the average normalized pressure $\mathrm{P}_{\mathrm{AVGN}}$ without belt with subjects lying on the back (left) and on the side (right). Average and standard deviation for the 16 subjects. 


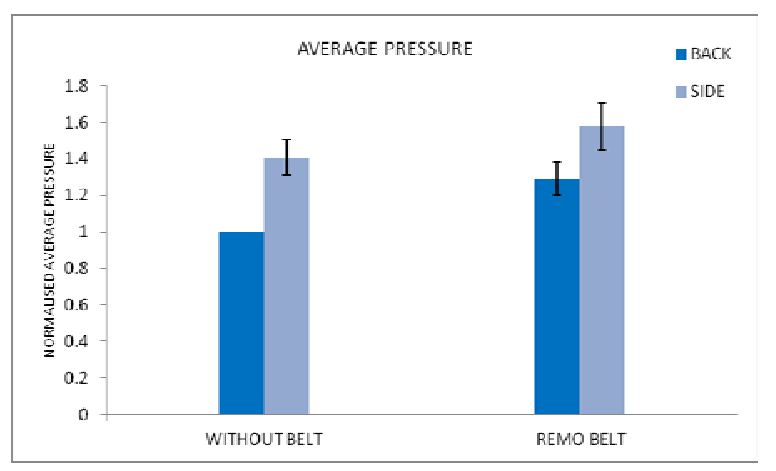

Fig. 3. Comparison of average normalised pressure $P_{\mathrm{AVGN}}$ with and without using the belt, with subjects lying on the back and on the side. Average and SD for 16 subjects.

Table 1

Comparison of average normalised pressure $\mathrm{P}_{\mathrm{AVGN}}$ with and without using the belt, with subjects lying on the back and on the side. Average and SD for 16 subjects.

\begin{tabular}{ccc}
\hline & \multicolumn{3}{c}{ Average normalised pressure (SD) [-] } \\
Case study & Back & Side \\
\hline Without belt & $1.00(0.0)$ & $1.41(0.12)$ \\
With belt & $1.29(0.09)$ & $1.57(0.14)$ \\
p-value & $<0.01$ & $<0.01$ \\
Diff (\%) & $\mathbf{2 9}$ & $\mathbf{1 1}$ \\
\hline
\end{tabular}

\subsection{Effect of belt size}

Fig. 4 and Table 2 show the average pressures $P_{\text {Avg }}$ for the 3 different belts used in the experiment. Number 1 corresponds to the smallest size, number 2 to the medium size and number 3 to the largest. The size of the belt used has no significant effect $(p>0.05)$ on the average pressure for both positions.

\subsection{Effect of belt on contact area}

The contact area was calculated for the cases where no belt was used and for each of the three different belt sizes. The two positions (lying on the back and on the side) were considered. The results are shown on Fig. 5 and summarised in Table 3. The belt has no significant influence $(\mathrm{p}>0.05)$ on the contact area for both positions.

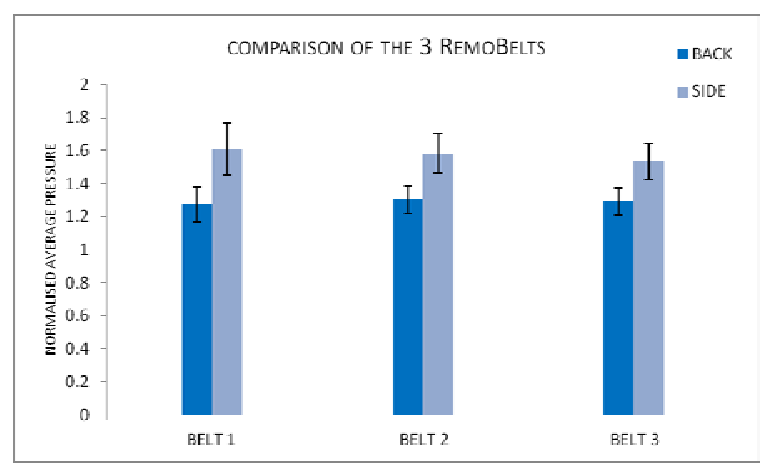

Fig. 4. Comparison of average normalised pressure $P_{\mathrm{AVGN}}$ for the 3 different sizes of belts, with subjects lying on the back and on the side. Average and SD for 16 subjects.

Table 2

Comparison of average normalised pressure $\mathrm{P}_{\mathrm{AVGN}}$ for the 3 different sizes of belts, with subjects lying on the back and on the side. Average and SD for 16 subjects.

\begin{tabular}{|c|c|c|}
\hline \multirow[b]{2}{*}{ Belt type } & \multicolumn{2}{|c|}{ Average normalised pressure (SD) [-] } \\
\hline & Back & Side \\
\hline Belt 1 & $1.28(0.11)$ & $1.61(0.16)$ \\
\hline Belt 2 & $1.30(0.08)$ & $1.58(0.13)$ \\
\hline Belt 3 & $1.29(0.08)$ & $1.53(0.12)$ \\
\hline
\end{tabular}

\subsection{Change in pressure over time}

For each trial, the average pressure on the contact area was calculated at each frame, allowing the change in pressure over time to be calculated. The values were normalised using the method described in section 2.3.2.

The duration of trials was 4 minutes. The change $\Delta \mathrm{P}$ of the average pressure over these 4 minutes was defined as:

$\Delta P=\frac{P_{E N D}-P_{S T A R T}}{P_{S T A R T}}$

Where $\mathrm{P}_{\text {start }}$ and $\mathrm{P}_{\text {end }}$ are the average pressures over the first 10 and the last 10 seconds of the trial, respectively.

Fig. 6 shows the values of $\Delta \mathrm{P}$, expressed as a percentage, for the cases where no belt was used, and when a belt was used (average of the 3 belts). The results are summarised in Table 4 . The pressure increase over time is significantly higher $(p<0.01)$ when no belt is used, for both positions. 


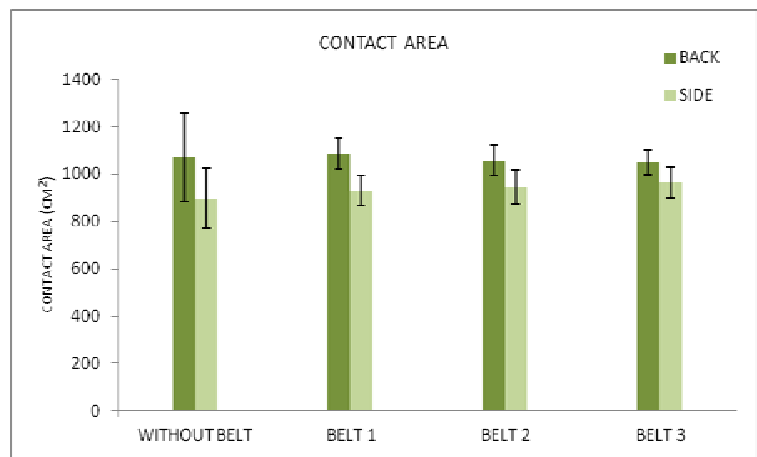

Fig. 5. Surface of contact area $(\mathrm{cm} 2)$ when using the 3 different sizes of belts, and when using no belt. Average and SD for 16 subjects.

Table 3

Surface of contact area $(\mathrm{cm} 2)$ when using the 3 different sizes of belts, and when using no belt. Average and SD for 16 subjects.

\begin{tabular}{lcc}
\hline \multicolumn{1}{c}{ Belt type } & \multicolumn{2}{c}{ Average contact surface (SD) $\left[\mathrm{cm}^{2}\right]$} \\
\hline without belt & Back & Side \\
Belt 1 & $1070(187)$ & $897(126)$ \\
Belt 2 & $1086(66)$ & $926(66)$ \\
Belt 3 & $1057(63)$ & $944(72)$ \\
\hline
\end{tabular}

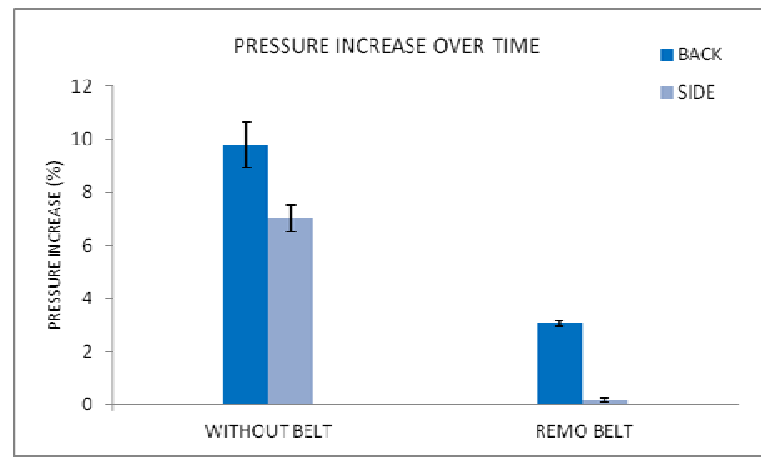

Fig. 6. Evolution of pressure over time (expressed as percentage of the initial pressure). Average and SD for 16 subjects.

Table 4

Evolution of pressure over time (expressed as percentage of the initial pressure). Average for 16 subjects.

\begin{tabular}{ccc}
\multicolumn{3}{c}{ 1nitial pressure). Average for 16 subjects. } \\
\hline & \multicolumn{3}{c}{$\Delta \mathrm{P}(\mathrm{SD})[\%]$} & Side \\
Back & $7.0(0.51)$ \\
Without belt & $9.8(0.87)$ & $0.2(0.09)$ \\
With belt & $3.1(0.11)$ & \\
\hline
\end{tabular}

\subsection{Effect of belt on pressure distribution}

The method described in 2.3.4 was used to determine the effect of the belt on the distribution of pressure. The results are presented in Fig. 7.

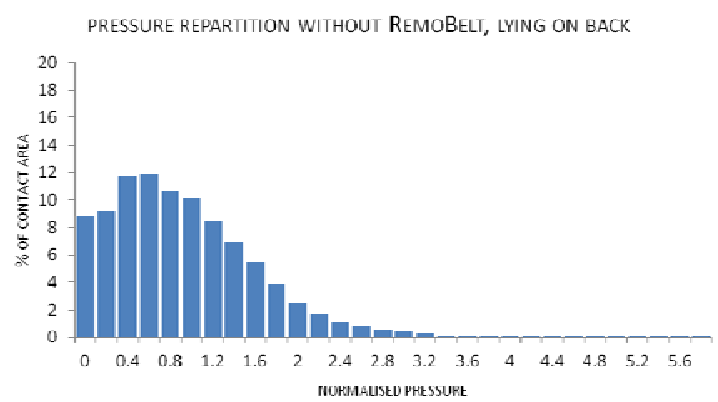

PRESSURE REPARTITION USING REMOBELT, LYING ON BACK

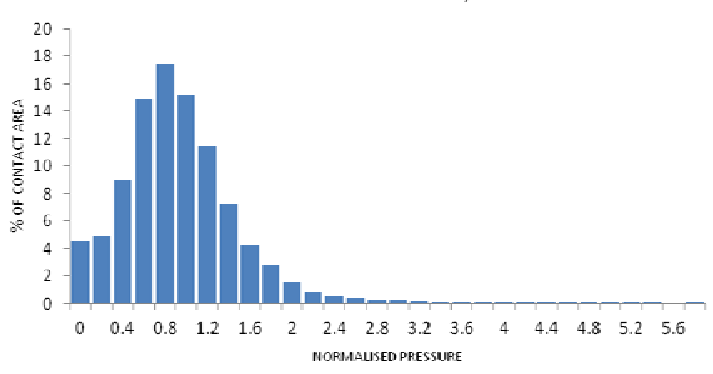

PRESSURE REPARTITION WITHOUT REMOBELI, LYING ON SIDE

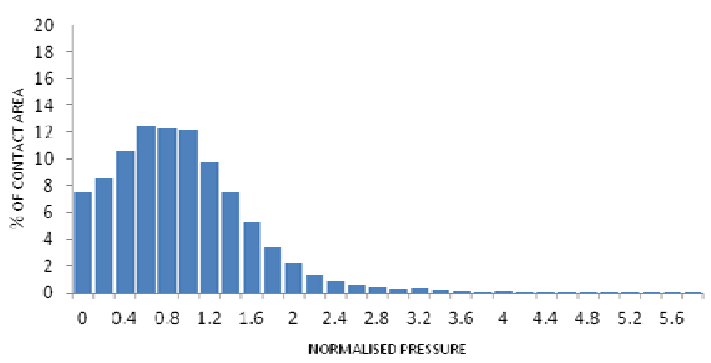

PRESSURE RFPARTITION USING RFMOBFLT, LYING ON SIDE

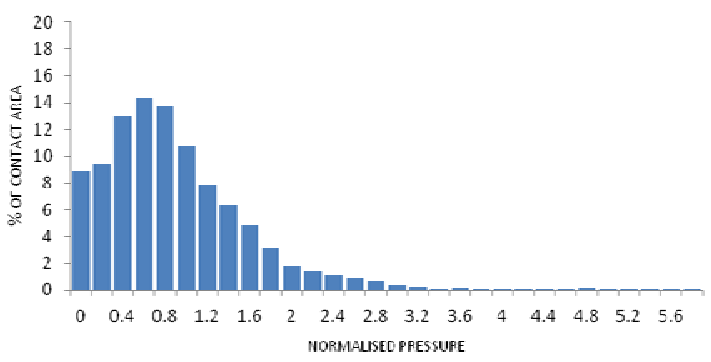

Fig. 7.Pressure distribution while lying on back and side, with and without belt. Average for 16 subjects. 


\section{Discussion and conclusion}

\subsection{Effect of the position without belt}

From Fig. 2b it can be seen that the average pressure is significantly higher when the subjects are lying on their side.

There are two reasons for this. Firstly, the contact area is reduced when lying on the side as can be seen from Fig. 5. Due to the same weight being supported on a smaller surface, the average pressure on that surface increases.

Secondly, the pressure is more evenly distributed when lying on the side (see Fig. 7). Results showed there are more pressure peaks when the subjects are lying on their back. These pressure peaks support a higher percentage of the subjects' weight resulting in the other zones of the contact area showing less pressure, hence a lower average when lying on the back.

Finally, looking at Fig. 6, it can be seen that the increase in pressure over time is higher when subjects are lying on the back. This is due to the fact that, over time, the deformation of the foam of the mattress tends to reduce the pressure peaks, and therefore the average pressure increases.

\subsection{Effect of using a belt}

\subsubsection{Average pressure}

It can be seen from Fig. 3 that the use of a belt tends to increase the average pressure significantly, independently of the position of the subject. We can also note that the size of the belt used has very little impact on the average pressure (Fig. 4).

This could be due to the fact that the use of a belt raises the buttocks region when lying on a bed, therefore a higher portion of the body weight has to be supported by this body part, thus increasing the pressure in this area.

Therefore, the only criterion to consider when choosing the size of the belt is the subject's size. The belt that best fits to the subject should be used.

\subsubsection{Contact area}

The Fig. 5 indicates that using a belt does not significantly change the surface of the contact area.

However, we can see that the surface of contact is less variable for different subjects when using the belt. This is due to the fact that the subject's anthropometry, and particularly Body Mass Index (BMI), has a greater influence on the contact area when no belt is used. As a result, the variation (SD) of the contact area between subjects is high. The use of the belt reduces this variability as the contact area is determined by the size of the belt itself which remains unchanged.

To summarise, the use of a belt tends to lower the contact area for tall and/or high BMI subjects and to increase it for short/low BMI subjects. This could be an important factor to consider for frail elderly with low BMI, because in this case increasing the contact area could lead to a significant pressure relief.

\subsubsection{Pressure distribution}

Fig. 7 shows the impact of the belt usage on the pressure distribution. It is clear that the belt greatly increases the evenness of pressure distribution. There are fewer pressure peaks, regardless of the lying position adopted.

However the pressure redistribution is higher when lying on the back. The reason for this difference is that there are fewer pressure peaks when lying on the side. As the pressure is already more evenly distributed when lying on the side, the pressure redistribution effect of the belt is lower.

\subsubsection{Evolution of pressure over time}

Fig. 6 and Table 4 show the change in pressure over time when the subject is lying on the back and on the side, respectively. We can see from the figures that there is an increase of the average pressure over time when no belt is employed, whereas no increase is observed when using a belt.

When lying on the back, the increase in pressure is more than 3 times lower when using a belt; this effect is even more pronounced when lying on the side where the increase is 35 times lower with the belt. The increase over time when using the belt is $0.2 \%$ which can be considered negligible.

The reasons for this are analogue to section 4.1: the more pressure peaks, the higher the average pressure increases over time. As using a belt nearly removes the peaks of pressure, it also influences the increase in pressure over time.

\subsection{Limitations}

One limitation with this study was that pressure measurements were only captured for a period of 4 minutes. However, the belt has been designed to be used for an extended period of time (several hours).

Secondly, the pressure distribution at the skin to belt interface was not measured due to technical limitations. The pressure at this interface might be differ- 
ent from the pressure at the belt to mattress interface. Although measuring the pressure at the skin/belt interface is technically challenging, it could give better insight on the effect of belt on pressure redistribution.

Another limitation was that pressure in the shoulder region was not measured. The use of the belt may have caused pressure in this region to increase due to the raised level of the buttocks/hips. Future studies should aim to investigate this.

Finally, only one mattress type has been used for this study. Although the mattress used is commonly found in hospital environments, investigating the effect of the belt on different types of mattresses, in particular pressure reducing mattresses, could give more insight into its potential applications.

\subsection{Future work}

A key finding was that the belt prevented an increase in pressure over time. Future work should be conducted to identify the differences that occur over extended periods of time. The pressure was still increasing without the use of the belt after a period of four minutes. Therefore, the differences may actually increase as time progresses. Further research should also measure pressure between the person and belt, not just the belt and hospital mattress. This may provide more detailed knowledge on how the pressures are influenced by the use of the belt.

Secondly, the subjects that took part in this experiment were relatively young. It could be interesting to perform this kind of study with elderly people, as they are a population more prone to developing pressure ulcers [1].

Lastly, pressure is only one of the factors that influence pressure sores. Other issues that also impact on pressure sores include shear forces, friction and humidity [2]. The impact of the belt on these other factors also needs to be tested in order to fully validate the product.

\subsection{Conclusion}

This study identified that higher peaks of pressure are evident when the subjects were lying on their back.

The use of the belt reduced pressure peaks and this was most evident when subjects were lying on their back. The belt therefore may achieve its purpose because previous studies have shown that pressure ulcers are associated with peak pressures [1].

There was an increased average pressure identified with the use of the belt. This could be due to the fact that the use of a belt raises the buttocks, therefore increasing the percentage of the body weight that is supported by this body region.

Another important finding was that the increase in pressure over the four minute period was reduced significantly through the use of the belt.

Future research should conduct trials over a longer period to identify the extent of these differences and aim to validate the belt across other factors which impact on pressure sores (e.g. friction and humidity).

\section{References}

[1] D.M. Brienza, P.E. Karg, M.J. Geyer, S. Kelsey and E. Trefler, The relationship between pressure ulcer incidence and buttock-seat cushion interface pressure in at-risk elderly wheelchair users, Archives of Physical Medicine and Rehabilitation, vol. 82, no. 4, 2001, pp. 529-533.

[2] M. Reddy, S.S. Gill and P.A. Rochon, Preventing pressure ulcers: A systematic review, Journal of American Medical Association, vol. 296, no. 8, 2006, pp. 974-984.

[3] L.J. Russell, T.M. Reynolds, C. Park, S. Rithalia, M. Gonsalkorale, J. Birch, D. Torgerson and C. Iglesias, Randomised clinical trial comparing 2 support surfaces: Results of the prevention of pressure ulcers study, Advances in Skin and Wound Care, vol. 16, no. 6, 2003, pp. 317-327. 